\title{
Correlation between short- and long-term effects of intravitreal ranibizumab therapy on macular edema after branch retinal vein occlusion: a prospective observational study
}

\author{
Yoshiro Minami ${ }^{1 *}$, Taiji Nagaoka², Akihiro Ishibazawa ${ }^{1,2}$ and Akitoshi Yoshida²
}

\begin{abstract}
Background: The correlation between the short- and long-term effects of intravitreal ranibizumab (IVR) on macular edema after branch retinal vein occlusion (BRVO) remains unclear. We assessed the correlation between the short- and long-term effects of IVR on macular edema after BRVO.

Methods: Twenty-one eyes with macular edema after BRVO were enrolled in this prospective observational study. We measured the foveal thickness (FT) and the best-corrected visual acuity (BCVA) before, 1 day after, and 1 month after IVR $(0.5 \mathrm{mg})$ and then at least every 2 months thereafter until 6 months after the injection. If the macular edema recurred, another injection was administered. The primary endpoint was the change from baseline in the BCVA $(\triangle V A)$.

Results: The mean logarithm of the minimum angle of resolution VA improved significantly $(p=0.01, p<0.0001$, respectively) after 1 day from $0.65 \pm 0.28$ to $0.51 \pm 0.21$ (20/89 to 20/63, Snellen equivalent) and after 6 months to 0 . $29 \pm 0.24(20 / 39$, Snellen equivalent). The mean FT decreased significantly $(p<0.0001)$ after 1 day from $482 \pm 85 \mu \mathrm{m}$ to $349 \pm 75 \mu \mathrm{m}$ and after 6 months to $305 \pm 84 \mu \mathrm{m}$. The 1-day VA was significantly $(r=0.68, p=0.0007)$ positively correlated with the 6-month $V A$. The 1 -day $\Delta V A$ was significantly $(r=0.79, p<0.0001)$ positively correlated with the 6month $\triangle \mathrm{VA}$.
\end{abstract}

Conclusions: The short-term effects of IVR may predict the long-term effects of IVR in macular edema secondary to BRVO.

Trial registration: Trial registration number: UMIN000027003. Retrospectively registered. (April/15/2017)

Keywords: Branch retinal vein occlusion, Macular edema, Ranibizumab

\section{Background}

Retinal vein occlusion (RVO), including branch RVO (BRVO), hemiretinal vein occlusion, and central RVO, and diabetic retinopathy are common retinal vascular diseases that cause moderate or severe visual loss in working-age patients [1-3]. RVOs have been estimated to develop in about 16 million people worldwide, and BRVO accounts for nearly $80 \%$ of those cases. The

\footnotetext{
* Correspondence: y37373m@yahoo.co.jp

${ }^{1}$ Department of Ophthalmology, Nayoro City General Hospital, Nishi 7

Minami 8-1, Nayoro 096-8511, Japan

Full list of author information is available at the end of the article
}

predominant cause of decreased visual acuity (VA) due to RVOs is macular edema when the fovea is involved [1-3]. Because the Branch Vein Occlusion Study Group reported the efficacy of grid laser treatment for macular edema after BRVO, it has been the standard therapy for treating macular edema associated with BRVO for about 30 years [4].

The Ranibizumab for the Treatment of Macular Edema following Branch Retinal Vein Occlusion: Evaluation of Efficacy and Safety (BRAVO) study found that ranibizumab (Lucentis, Novartis, Basel, Switzerland), a humanized affinity-matured vascular endothelial growth 
factor (VEGF) antibody fragment that specifically binds all isoforms of VEGF-A, clearly improved macular edema due to BRVO $[5,6]$. We recently reported the short-term effect of intravitreal ranibizumab (IVR) injections on macular edema secondary to BRVO, and the short-term effect (within a day) was correlated with the therapeutic efficacy 1 week and 1 month after the IVR injection [7]. However, the correlation between the short- and long-term effects of IVR injections on macular edema due to BRVO is undetermined. In the current study, we report the short-term (1 day) and the longterm (6 months) effects and the correlation between them.

\section{Methods}

\section{Subjects}

The study adhered to the tenets of the Declaration of Helsinki and followed the guidelines approved by the ethics committee of our institution. All patients were treatment-native Japanese individuals who provided informed consent before participation in the study. The study was performed at Nayoro City General Hospital between October 2013 and April 2016. The inclusion criteria were the presence of macular edema that involved the fovea after development of BRVO before therapy (foveal thickness [FT] at baseline $\geqq 300 \mu \mathrm{m}$ ), no history of IVR injection, and no history of other treatments for macular edema within the previous 3 months. Application of photocoagulation to the unvascularized area outside of the vessel arcade before and throughout the follow-up period was permitted. No patients were treated with a dexamethasone implant because the treatment had not yet been approved in Japan. The exclusion criteria were the presence of other retinal diseases such as diabetic retinopathy and age-related macular degeneration. Patients diagnosed with hemicentral vein occlusion were considered to have BRVO according to the BRAVO study criteria [5]. The patients underwent comprehensive ophthalmologic examinations including measurement of the best-corrected visual acuity (BCVA), slit-lamp biomicroscopy with a noncontact fundus lens, measurement of intraocular pressure (IOP) with noncontact tonometers, and spectral-domain optical coherence tomography (SD-OCT) (RetinaScan RS-3000, Nidek, Gamagori, Japan). The BCVA was measured using a standard Japanese decimal VA chart at $5 \mathrm{~m}$. The decimal values were converted to the logarithm of the minimum angle of resolution (logMAR) units for statistical analyses. To evaluate the FT, the macular map analysis protocol of the RS-3000 SD-OCT was used. The FT was defined as the average of all points in the inner circle (radius, $1 \mathrm{~mm}$ ) at the center of the nine sectors defined by the Early Treatment Diabetic Retinopathy Study grid [8].

\section{IVR injection}

IVR injections were administered according to a sterile technique $(0.5 \mathrm{mg} / 0.05 \mathrm{~mL})$ using a 30 -gauge needle. Before injection, anterior chamber paracentesis was performed using a 27-gauge needle to prevent IOP increases. Topical antibiotics were instilled prophylactically for 1 week after the IVR injection.

\section{Time course of evaluation of the therapeutic effect of IVR injections and criteria for additional injections}

The FT and BCVA were measured before the IVR injection (baseline) and 1 day after, 1 month after, and at least every 2 months (1-2 months) after the IVR injection until 6 months. If recurrent macular edema was detected, another injection was administered. Recurrent macular edema was defined as an increase in the FT exceeding $50 \mu \mathrm{m}$ over a previous measurement. Patients who did not present at the time of examination or did not receive another injection despite recurrent macular edema were excluded from the current study. The changes in the logMAR VA $(\triangle \mathrm{VA})$ from baseline were calculated as $\triangle \mathrm{VA}-1$ day and $-1,-3$, and -6 months. The changes in the FT $(\Delta \mathrm{FT})$ from baseline also were calculated as $\Delta$ FT- 1 day, $-1,-3$, and -6 months.

\section{Data analysis}

All values are expressed as the means \pm standard deviations. Overall differences in the logMAR VA and the FT between baseline and 1 day and 1,3, and 6 months after the IVR injections were assessed using repeated measures analysis of variance. Subgroup-specific changes in the VA and FT were analyzed using Dunnett's multiple comparisons test. To determine if the early effectiveness of IVR injections predicts the late-phase outcome after the injections, the correlations between the VA at 1 day and the VA at 6 months and between the $\triangle \mathrm{VA}-1$ day and the $\triangle \mathrm{VA}-6$ months and between the $\Delta \mathrm{FT}-1$ day and the $\triangle$ VA-6 months were evaluated using Pearson's correlation model and linear regression analysis. To investigate the correlations between the symptom duration and the VA at 6 months or the $\triangle$ VA- 6 months, Spearman's rank correlation coefficient was used because the symptom duration was wide. $P<0.05$ was considered significant.

\section{Results}

Table 1 shows the patient baseline characteristics. Thirty-five consecutive eyes of 34 patients with macular edema due to BRVO who received the first IVR injection during this study were included. Fourteen eyes of 13 patients were lost to follow-up during the study. Thus, 21 eyes of 21 patients were analyzed. The mean number of injections was $2.0 \pm 0.59$ during this study. Two eyes underwent photocoagulation before study entry and two 
Table 1 Baseline Characteristics of 21 Patients (21 Eyes) with Macular Edema after BRVO

\begin{tabular}{ll}
\hline Age (years, mean \pm SD) & $71.0 \pm 10.3$ \\
\hline Gender (male/female) & $6 / 15$ \\
Symptom duration (months, mean \pm SD) & $9.4 \pm 11.2$ \\
History of photocoagulation (eyes) & $2(10 \%)$ \\
BCVA (logMAR, mean \pm SD) & $0.65 \pm 0.28$ \\
(Snellen equivalent) & $20 / 89$ \\
Baseline FT ( $\mu$ m, mean \pm SD) & $482 \pm 85$ \\
Phakic eye (eyes) & $19(90 \%)$ \\
Systemic hypertension (patients) & $11(52 \%)$ \\
Diabetes mellitus (patients) & $0(0 \%)$
\end{tabular}

BRVO branch retinal vein occlusion, logMAR logarithm of the minimum angle of resolution, $S D$ standard deviation, $B C V A$ best-corrected visual acuity, $F T$ foveal thickness

eyes underwent photocoagulation of non-perfused areas outside of the vessel arcade during this study. Eleven patients had systemic hypertension and no patients had diabetes mellitus. Two eyes had an intraocular lens implanted previously. The average time from the development of BRVO to the IVR injection was $9.4 \pm 11.2$ months. Ocular hypertension $(>21 \mathrm{mmHg}$ ) or a drastic IOP elevation (elevation more than $5 \mathrm{mmHg}$ from baseline) did not occur at any time points. No treatment complications such as endophthalmitis or retinal detachment developed during the study.

Figure 1 shows the mean changes in the logMAR VA after the IVR injection. The mean logMAR VA improved significantly on 1 day and 1,3 , and 6 months $(p=0.01$, $p<0.0001, p<0.0001$, and $p<0.0001$, respectively) from the baseline values of $0.65 \pm 0.28$ (20/89, Snellen equivalent) to $0.51 \pm 0.21$ (20/63 Snellen equivalent), $0.35 \pm 0.22$ (20/45, Snellen equivalent), $0.31 \pm 0.25(20 /$

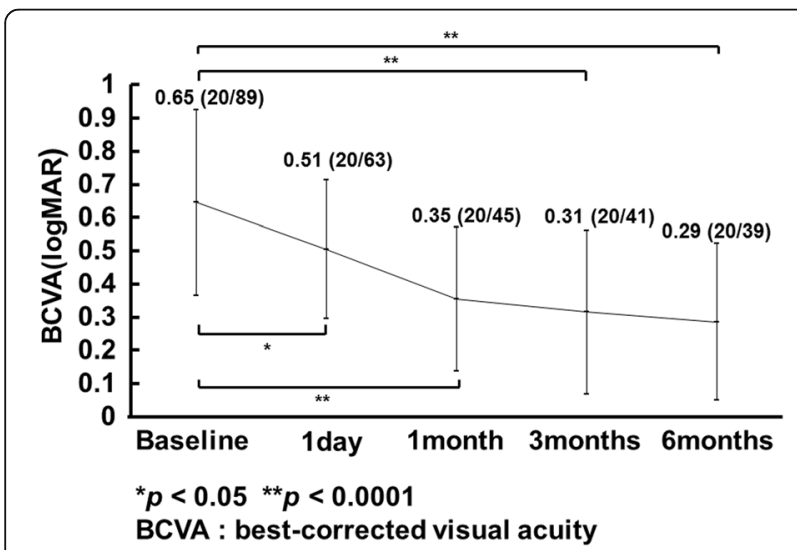

Fig. 1 The mean changes in the logarithm of the minimum angle of resolution (logMAR) best-corrected visual acuity (BCVA) at each follow-up evaluation ( $n=21$ eyes). The values are expressed as the mean \pm standard deviation (Snellen equivalent). The BCVA is improved significantly from baseline $\left({ }^{*} p<0.05,{ }^{* *} p<0.0001\right)$
41, Snellen equivalent), and $0.29 \pm 0.24$ (20/39, Snellen equivalent) after the IVR injections. The mean FT decreased significantly $(p<0.0001)$ from the baseline value of $482 \pm 85 \mu \mathrm{m}$ to $349 \pm 75 \mu \mathrm{m} 1$ day after the IVR injection. Significant reductions also were seen to $263 \pm 39,319 \pm 96$, and $305 \pm 84 \mu \mathrm{m}$ at 1,3 , and 6 months, respectively ( $p<0.0001$ at all time points). The BCVA 1 day after the IVR injection was correlated significantly $(r=0.68, p=0.0007)$ with the BCVA 6 months after the IVR injection (Fig. 2a). The $\triangle$ VA1 day was correlated significantly $(r=0.79, p<0.0001)$ with the $\triangle$ VA-6 months (Fig. 2b). The $\triangle$ FT-1 day was not correlated significantly $(r=0.09, p=0.68)$ with the $\triangle$ VA-6 months. The symptom duration was correlated significantly $(p=0.003)$ with the BCVA 6 months after the IVR injection but not with the $\triangle$ VA- 6 months $(p=0.12)$. The $\Delta \mathrm{VA}-1$ day was correlated significantly $(r=0.67, p=0.001)$ with the baseline VA (Fig. 3).

\section{Discussion}

In the current study, the BCVA and FT improved significantly 1 day after the IVR injection from the baseline values, and those improvements were sustained 6 months after the first IVR injection (Fig. 1). We recently reported that the BCVA and FT improved significantly from the baseline values within 1 day after the IVR injection in patients with macular edema secondary to BRVO [7]. In the current study, we showed that the BCVA 1 day after the IVR injection was correlated with the BCVA 6 months after the IVR injection in patients with macular edema due to BRVO (Fig. 2a) and the $\triangle \mathrm{VA}-1$ day was correlated more significantly with the $\Delta$ VA-6 months (Fig. 2b). This indicated that we can predict the long-term (6-month) functional outcome based on the short-term (within 1 day) functional effect of the IVR injections. In contrast, the $\triangle \mathrm{FT}-1$ day was not correlated significantly with the $\triangle \mathrm{VA}-6$ months, suggesting that it is difficult to predict the long-term functional effect based on the short-term structural change after the IVR injections. Although the baseline BCVA was reported to be predictive of the long-term effect of IVR injections [9], the baseline BCVA was not correlated significantly $(p=0.09)$ with the BCVA 6 months after the first IVR injection in the current study. However, in the current study, the BCVA 1 day after the IVR injection was correlated significantly with the BCVA at 6 months after the IVR injection and the $\triangle \mathrm{VA}-1$ day was correlated significantly with the $\triangle$ VA-6 months (Fig. 2b). Our results suggested that measuring the BCVA 1 day after the IVR injection might be a better predictive factor of the long-term effect of IVR injections on the macular edema secondary to BRVO.

The symptom duration has been reported to be predictive of the efficacy of anti-VEGF therapy on the 

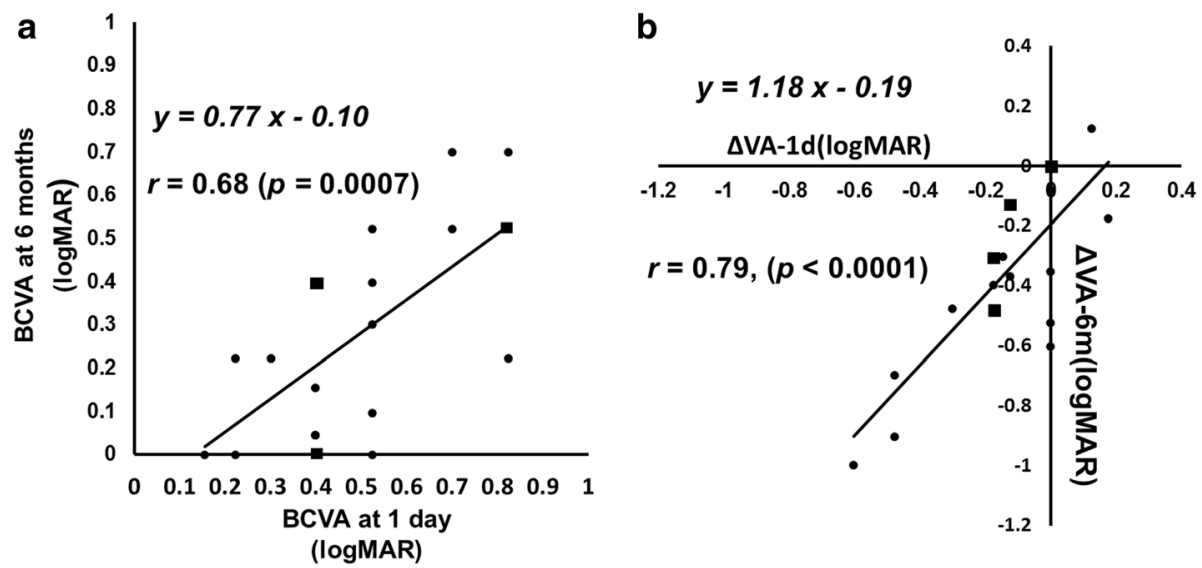

Fig. 2 a The relationship between the changes in the logarithm of the minimum angle of resolution (logMAR) best-corrected visual acuity (BCVA) 1 day after the first intravitreal ranibizumab (IVR) injection and the BCVA 6 months after the first IVR injection. There is a significant ( $r=0.68$, $p=0.0007)$ positive correlation between them. The squares indicate that two eyes overlap. $\mathbf{b}$ The relationship between the changes in the I ogMAR BCVA from baseline to 1 day ( $\triangle \mathrm{VA}-1$ day) and the changes in the BCVA from baseline to 6 months ( $\triangle \mathrm{VA}-6$ months) after the IVR injections. There is a significant $(r=0.79, p<0.0001)$ positive correlation between them. The squares indicate that two eyes overlap

macular edema secondary to BRVO $[9,10]$. In the current study, the symptom duration was correlated significantly with the BCVA 6 months after the first IVR injection. However, the symptom duration was not correlated significantly with the baseline BCVA. The current results suggested that we can obtain better long-term effects of the IVR injections when the first injection is administered sooner after the onset of BRVO.

Although the result might be affected by the protocol for the therapy, the best protocol for an IVR injection to treat BRVO remains controversial, i.e., monthly consecutive injections (mostly for 3 months) or pro re nata (PRN) injections. As reported in the BRAVO study $[5,6]$ and

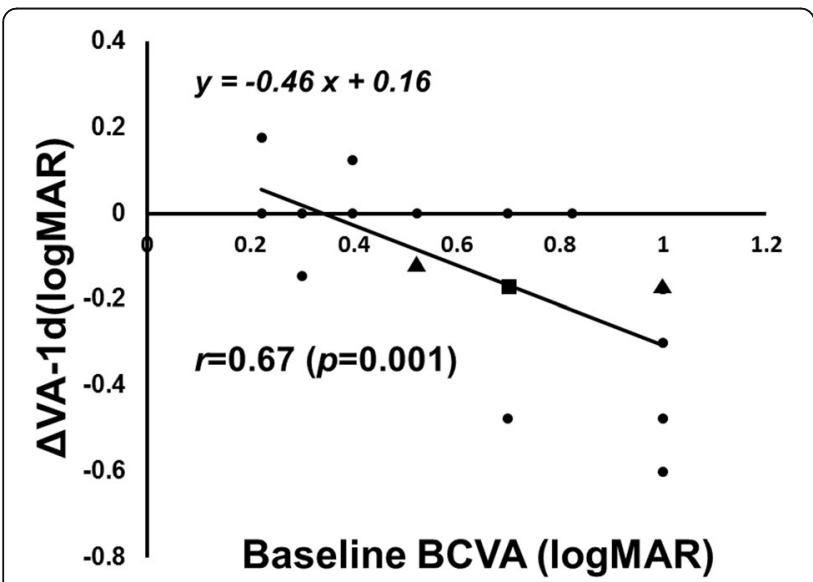

Fig. 3 The relationship between the changes in the logarithm of the minimum angle of resolution best-corrected visual acuity (logMAR BCVA) from baseline to 1 day ( $\triangle \mathrm{VA}-1$ day) and the baseline VA. There is a significant ( $r=0.67, p=0.001)$ positive correlation between them. The squares indicate that two eyes overlap; the triangles indicate that three eyes overlap other clinical studies [11, 12], consecutive IVR injections are effective for treating the macular edema associated with BRVO. However, several authors have reported the efficacy of PRN intravitreal bevacizumab (IVB) (Avastin, Genentech Inc., South San Francisco, CA) and IVR injections [9, 10, 13, 14]. Ito et al. [15] reported that the group treated with PRN IVB injections for macular edema secondary to BRVO achieved similar visual outcomes with fewer injections compared with the group treated with three consecutive IVB injections. Therefore, it is important to achieve an effectiveness level similar to that of IVR injections with fewer injections, and we believe that PRN injections are the best protocol for IVR therapy to treat macular edema secondary to BRVO. Therefore, in the current study, we followed a PRN injection protocol.

The current study had some limitations. First, the number of patients was too small for subgroup analysis. Because the prognosis for the VA recovery was reported to be affected by the duration of the macular edema and previous treatment before the IVB injection [10], we excluded patients who had been treated previously for macular edema within 3 months of study entry. We previously reported that the BCVA and FT improved significantly from the baseline within 1 day after the IVR injection in patients with macular edema secondary to BRVO in 23 eyes [7]. Moreover, we also revealed that the $\triangle$ VA-1 day was correlated significantly with the $\triangle$ VA-1 month. Although the number of eyes was small, the current result regarding the relationship between the $\Delta$ VA-1 day and $\triangle$ VA-1 month was similar to our previous report [7]. Second, because the current study had no control group, we could not exclude the effect of spontaneous regression on the effect of the IVR injections on macular edema. Macular edema secondary to BRVO has 
a high level of spontaneous regression within 3 months of the occurrence [4]. We could not exclude the effect of spontaneous regression on our results. Indeed, seven current patients received an IVR injection less than 3 months after the occurrence of BRVO. We confirmed that the current results (significant correlation between VA at 1 day and VA at 6 months after the IVR injection and between the $\triangle \mathrm{VA}-1$ day and $\triangle \mathrm{VA}-6$ months) were unchanged after the data from those seven patients were removed from the analysis (data not shown). Taken together, we speculated that the effect of spontaneous regression had little effect on the current results. In addition, previous major clinical studies have included patients in whom macular edema developed within 3 months before the first IVR injection.

\section{Conclusion}

In conclusion, the current findings suggested that the effects of IVR injections might be detectable as early as 1 day after treatment and evaluation of the short-term effects of IVR injections can predict the long-term outcome of IVR injections administered to treat macular edema secondary to BRVO.

\begin{abstract}
Abbreviations
BCVA: Best-corrected VA; BRVO: Branch retinal vein occlusion; FT: Foveal thickness; IVB: Intravitreal injection of bevacizumab; IVR: Intravitreal ranibizumab; logMAR: Logarithm of the minimum angle of resolution: PRN: Pro re nata; RVO: Retinal vein occlusion; SD-OCT: Spectral-domain optical coherence tomography; VA: Visual acuity; VEGF: Vascular endothelial growth factor; $\triangle \mathrm{FT}$ : Changes in the FT; $\triangle \mathrm{VA}$ : Changes in the logMAR VA
\end{abstract}

Acknowledgements

Not applicable.

\section{Funding}

No funding was obtained for this study.

\section{Availability of data and materials}

The datasets during and/or analyzed during the current study are available from the corresponding author on reasonable request.

\section{Authors' contributions}

YM and Al were responsible for collection of data. YM and TN performed the statistical analysis. YM, TN, Al, and AY were responsible for interpretation of results. YM participated in the design and wrote the first draft of the manuscript. All authors reviewed and approved the final manuscript.

\section{Competing interests}

The authors declare that they have no competing interests.

\section{Consent for publication}

Not applicable.

\section{Ethics approval and consent to participate}

The ethics committee of Nayoro City General Hospital approved this study. Nayoro city general hospital ethics committee. Reference number 187.

\section{Publisher's Note}

Springer Nature remains neutral with regard to jurisdictional claims in published maps and institutional affiliations.

\section{Author details}

'Department of Ophthalmology, Nayoro City General Hospital, Nishi 7 Minami 8-1, Nayoro 096-8511, Japan. ${ }^{2}$ Department of Ophthalmology, Asahikawa Medical University, Asahikawa, Japan.

Received: 18 December 2016 Accepted: 8 June 2017

Published online: 13 June 2017

\section{References}

1. Klein R, Klein BE, Moss SE, et al. The epidemiology of retinal vein occlusion: the beaver dam eye study. Trans Am Ophthalmol Soc. 2000;98:133-41. discussion 141-133

2. Rogers S, Mclntosh RL, Cheung N, et al. The prevalence of retinal vein occlusion: pooled data from population studies from the United States, Europe, Asia, and Australia. Ophthalmology. 2010;117:313-9. e311

3. Rogers SL, McIntosh RL, Lim L, et al. Natural history of branch retinal vein occlusion: an evidence-based systematic review. Ophthalmology. 2010;117:1094-101. e1095

4. Argon laser photocoagulation for macular edema in branch vein occlusion. The Branch Vein Occlusion Study Group. Am J Ophthalmol. 1984;98:271-282.

5. Campochiaro PA, Heier JS, Feiner $L$, et al. Ranibizumab for macular edema following branch retinal vein occlusion: six-month primary end point results of a phase III study. Ophthalmology. 2010;117:1102-12. e1101

6. Brown DM, Campochiaro PA, Bhisitkul RB, et al. Sustained benefits from ranibizumab for macular edema following branch retinal vein occlusion: 12month outcomes of a phase III study. Ophthalmology. 2011;118:1594-602.

7. Minami Y, Nagaoka T, Ishibazawa A, et al. Short-term effect of intravitreal ranibizumab therapy on macular edema after branch retinal vein occlusion. Retina. 2016;36:1726-32.

8. Classification of diabetic retinopathy from fluorescein angiograms. ETDRS report number 11. Early Treatment Diabetic Retinopathy Study Research Group. Ophthalmology. 1991;98:807-822.

9. Tadayoni R, Waldstein SM, Boscia F, et al. Individualized stabilization criteriadriven ranibizumab versus laser in branch retinal vein occlusion. Ophthalmology. 2016;123:1332-44.

10. Jaissle $G B$, Szurman $P$, Feltgen $N$, et al. Predictive factors for functional improvement after intravitreal bevacizumab therapy for macular edema due to branch retinal vein occlusion. Graefes Arch Clin Exp Ophthalmol. 2011:249:183-92.

11. Sophie R, Hafiz G, Scott AW, et al. Long-term outcomes in ranibizumabtreated patients with retinal vein occlusion; the role of progression of retinal nonperfusion. Am J Ophthalmol. 2013;156:693-705.

12. Brynskov T, Kemp H, Sorensen TL. Intravitreal ranibizumab for retinal vein occlusion through 1 year in clinical practice. Retina. 2014;34:1637-43.

13. Chung EJ, Hong YT, Lee SC, et al. Prognostic factors for visual outcome after intravitreal bevacizumab for macular edema due to branch retinal vein occlusion. Graefes Arch Clin Exp Ophthalmol. 2008;246:1241-7.

14. Gallego-Pinazo R, Dolz-Marco R, Pardo-Lopez D, et al. Ranibizumab for serous macular detachment in branch retinal vein occlusions. Graefes Arch Clin Exp Ophthalmol. 2013;251:9-14.

15. Ito Y, Saisin Y, Sawada O, et al. Comparison of single injection and three monthly injections of intravitreal bevacizumab for macular edema associated with branch retinal vein occlusion. Clin Ophthalmol. 2015;9:175-80.

Submit your next manuscript to BioMed Central and we will help you at every step:

- We accept pre-submission inquiries

- Our selector tool helps you to find the most relevant journal

- We provide round the clock customer support

- Convenient online submission

- Thorough peer review

- Inclusion in PubMed and all major indexing services

- Maximum visibility for your research

Submit your manuscript at www.biomedcentral.com/submit
C Biomed Central 